$\mathrm{UM}-\mathrm{TH} / 97-18$

hep-ph/9710487

\title{
On Non Perturbative Corrections to the Potential for Heavy Quarks
}

\author{
R. Akhoury and V.I.Zakharov \\ Randall Laboratory of Physics \\ University of Michigan \\ Ann Arbor, Michigan 48109-1120
}

\begin{abstract}
We discuss non perturbative corrections to the Coulomb-like potential of heavy quarks at short distances. We consider both the standard framework provided by infrared renormalons and the assumption that confinement does not allow weak fields to penetrate the vacuum. In the former case the leading correction at short distances turns out to be quadratic in $r$ for static quarks. In the latter case we find a potential which is proportional to $r$ as $r \rightarrow 0$. We point out that similar effects arise due to a new kind of non perturbative correction proportional to $1 / Q^{2}$, which is unaccounted for by the operator product expansion and which was recently discussed within a different framework. Phenomenological implications of the linear correction to the potential are briefly reviewed.
\end{abstract}

PACS numbers: 12.38.Cy, 12.39.Hg, 13.20.He 
1. Analytic studies of non-perturbative effects in QCD are limited by the fact that there does not exist a systematic procedure to account for confinement. We may attempt, however, to sort out and to parametrize its effects by considering the case when the perturbative part is dominant and the infrared sensitive contributions appear only as small power corrections in a large mass scale $Q$. Well known examples of this kind are provided by the applications of the operator product expansion such as the QCD sum rules [1]. More recently, renormalons [2] have emerged as a more universal tool for enumerating the power-like corrections even in situations where the operator product expansion does not apply ( for furthur references and review, see e.g., [3]).

In this note we will apply these techniques to the short-distance interaction between heavy quarks, $Q, \bar{Q}$. As is well known, this interaction is dominated by a Coulomb-like potential:

$$
\lim _{r \rightarrow 0} V(r)=-\frac{C_{F} \alpha_{s}(r)}{r}
$$

where the coefficient $C_{F}=4 / 3$ refers to the color-singlet channel. We will argue below that the character of the leading power corrections depend on the properties of confinement. In particular, the standard renormalon technique implies the effect of confinement to become important only at distances $R_{c r} \sim \Lambda_{Q C D}^{-1}$ and the leading power correction to (1) turns to be:

$$
\lim _{r \rightarrow 0} \delta V(r)_{\text {renormalon }}=c_{2} r^{2} .
$$

Similar results for the static potential have already been obtained in the literature by various techniques [4, 5, 6].

Although infrared renormalons probably correctly indicate the presence of some of the power corrections, it is an open questions whether they exhaust all the possibilities. In case of the static potential, infrared renormalons do not give any indication to a formation of a string at large distances and could miss, therefore, the leading correction at short distances as well. In fact, we will consider the power corrections to the static potential (1) in an alternative model which implements the assumption that the effects of confinement become important once the color fields become weak. In such a case (see below ) we find in contrast to Eq. (2), a leading correction which is linear in $\mathrm{r}$ :

$$
\lim _{r \rightarrow 0} \delta V(r)_{\text {non-standard }}=c_{1} r
$$

A crucial element to ensure such corrections appears to be the existence of small size non perturbative fluctuations in QCD.

Power corrections which go beyond the OPE and infrared renormalons have recently been highlighted both within a general dispersion approach [7, 8] and the ultravioletrenormalon technique [9]. Most remarkably, indications from lattice simulations for the existence of such corrections have very recently been obtained in 10. More specifically, one argues that the effective coupling may contain terms proportional to $1 / Q^{2}$ :

$$
\alpha_{e f f}\left(Q^{2}\right)=\alpha_{e f f}^{\text {pert. }}\left(Q^{2}\right)+c_{Q^{2}} \frac{\Lambda_{Q C D}^{2}}{Q^{2}}
$$

while infrared renormalons only indicate the presence of corrections proportional to $1 / Q^{4}$, corresponding to the gluon condensate. 
It is amusing to observe that the $1 / Q^{2}$ piece in (4) also would give rise to a linear correction to the potential. Thus it is interesting to speculate that the mechanism of the repulsion of weak fields by the vacuum could be a possible mechanism responsible for the correction (4) if its existence [10] is confirmed. We would like to emphasize that although the static potential is not directly observable, it can be measured on the lattice (for a recent study including further references see [11]). In particular, although the existence of a linear potential at large distances is well established and there is no indication to its change to the $r^{2}$ behaviour as predicted by (2), much more precise data is needed to either confirm or reject the presence of a linear term at short distances. On the other hand the linear correction Eq. (3) would also affect the by-now-standard theory of the bound states of heavy quarks (for a review and further references see, e.g., [12) and we will address this issue as well.

2. To evaluate the renormalon contribution to $V(r)$ consider first the one gluon exchange potential

$$
V(r)=-C_{F} \int d^{3} \mathbf{k}\left(4 \pi \alpha_{s}\left(\mathbf{k}^{2}\right)\right) \frac{\exp (i \mathbf{k} \cdot \mathbf{r})}{\mathbf{k}^{2}}
$$

where the leading logarithmic corrections have been incorporated into the running coupling constant, $\alpha_{s}\left(\mathbf{k}^{2}\right)$, and the fact that for static quarks it depends on $\mathbf{k}^{2}$ is crucial for further argument. Beyond the leading logs, Eq. (5) holds in an Abelian case and in the limit of large $N_{f}$ in QCD [6]. To find the renormalon contribution we write

$$
\alpha_{s}\left(\mathbf{k}^{2}\right)=\int d \sigma\left(\frac{k}{\Lambda_{Q C D}}\right)^{-2 \sigma b_{0}}
$$

whee $b_{0}$ is the first coefficient in the expansion of the $\beta$-function (for simplicity we confine ourselves to a one-loop $\beta$-function), and $\Lambda_{Q C D}$ is the position of the Landau pole.

Next, we substitute (6) into (5) and perform the integration over directions of $\mathbf{k}$ to get

$$
V(r)=-\frac{16 \pi}{3} \int_{0}^{\infty} d \sigma \int_{0}^{\infty} d k \frac{\sin (k r)}{k r}\left(\frac{k}{\Lambda_{Q C D}}\right)^{-2 \sigma b_{0}}
$$

Moreover, and since we are interested in $V(r)$ at small $r$, we expand in $k r$ :

$$
V(r)=-\frac{16 \pi}{3} \int_{0}^{\infty} \Lambda_{Q C D}^{2 \sigma b_{0}} d \sigma \int d k\left(k^{-2 \sigma b_{0}}+k^{2-2 \sigma b_{0}} r^{2}+\ldots\right),
$$

The renormalon poles at $\sigma=1 / 2 b_{0}, 3 / 2 b_{0} \ldots$ are clearly seen in the above and this suggests a modification of the Coulomb potential at small $r$ of the form:

$$
\delta V(r)=c_{0} \Lambda_{Q C D}+c_{2} \Lambda_{Q C D}^{3} r^{2}+\ldots
$$

where $c_{0,2}$ are constants and it is noteworthy that they do not contain any small coupling. This has already been noted in [6. Since we expand in $k r$ and $k \sim \Lambda_{Q C D}$ at the renormalon poles, Eq. (9) is valid at distances small compared to the confinement radius:

$$
r \ll \Lambda_{Q C D}^{-1}
$$


This result has been obtained via a Borel transformation to conform to the current usages in renormalon analysis. It may also be seen by noting that the pole at $k=\Lambda_{Q C D}$ in the standard, leading log expression for $\alpha_{s}$,

$$
\alpha_{s}\left(\mathbf{k}^{2}\right)=\frac{1}{b_{0} \log k^{2} / \Lambda_{Q C D}^{2}}
$$

implies that the Fourier integral above is undefined. According to the general theory of singular integrals, we may write

$$
\alpha_{s}\left(\mathbf{k}^{2}\right)=\mathrm{P} . \mathrm{P} \cdot \frac{1}{b_{0} \log k^{2} / \Lambda_{Q C D}^{2}}+\mathrm{i} c \Lambda_{Q C D}^{2} \delta\left(k^{2}-\Lambda_{Q C D}^{2}\right),
$$

where $c$ is an arbitrary constant. When integrated the last term, $\mathrm{i} c \Lambda_{Q C D}^{2} \delta\left(k^{2}-\Lambda_{Q C D}^{2}\right)$, is easily seen to give a correction to the potential,

$$
\delta V(r)=c \frac{\sin \left(r \Lambda_{Q C D}\right)}{r}
$$

which, upon expansion, reproduces the type of corrections given in Eq. (9).

Eq. (9) remains qualitatively unchanged upon inclusion of higher loop corrections as well. The results of explicit calculations (which are known now up to three loops [13]) are usually represented as an expansion, in $\alpha_{\overline{M S}}$ :

$$
V\left(\mathbf{k}^{2}\right)=-C_{F} \frac{4 \pi \sum a_{n} \alpha \frac{n}{M S}\left(\mathbf{k}^{2}\right)}{\mathbf{k}^{2}} .
$$

It is straightforward to see that renormalon contribution associated with higher powers of $\alpha\left(\mathbf{k}^{2}\right)$ give the same type of power corrections as above. Indeed, in the approximation of the one-loop $\beta$-function,

$$
\alpha^{2}\left(\mathbf{k}^{2}\right)=\frac{1}{2 b_{0}} \Lambda_{Q C D} \frac{d}{\Lambda_{Q C D}} \alpha\left(\mathbf{k}^{2}\right) .
$$

Applying the differentiation with respect to $\Lambda_{Q C D}$ at the last step, i.e. to Eqs. (9, 13) we immediately see that the Fourier transform of the $\alpha^{2}\left(\mathbf{k}^{2}\right)$ term brings the same kind of power corrections as above. This is true for higher powers of $\alpha\left(\mathbf{k}^{2}\right)$ as well so that Eq. (9) is reproduced by renormalons associated with any order in $\alpha\left(\mathbf{k}^{2}\right)$ in Eq. (14). Finally, inclusion of higher loops in the $\beta$-function itself is known 14 to modify the renormalon poles to renormalon cuts, the position of the singularity remaining unchanged. This would bring powers of logs in the correction to the potential but would not change the power of $r, r^{2}$.

It is worth pointing out that the renormalon contribution could be tried also at large distances. The resulting potential does not reproduce the linear rise at large $r$ and is readily seen to be devoid of physical meaning. The reason is that renormalons are a pure perturbative construct and do not produce any hint that at large $r$ a string is formed. This failure demonstrates a limitation of the renormalon technique - the existence of the Landau pole in the infrared region does not automatically imply confinement.

To go beyond the perturbative expansion (14) for $V(r)$ in the non- Abelian case one has to consider the Wilson loop average $\langle W(C)\rangle$ for the stretched rectangle $C=r \times T$ with small $r$ and large $T$ :

$$
V(r)=-\lim _{T \rightarrow \infty} \frac{1}{i T} \ln <\operatorname{Tr} \operatorname{Pexp}\left(i g \oint_{C} d x_{\mu} A_{a}^{\mu} T^{a}\right)>.
$$


While there is no rigorous way to evaluate $\langle W(C)>$ analytically, model calculations [4, 5] do reproduce the same $r^{2}$ correction as the leading one at short distances. In particular a model of Ref. [4] gives the following result:

$$
V(r)=-C_{F} \frac{\alpha(r)}{r}+r^{2} \Phi(r)+O\left(r^{4}\right)
$$

where

$$
\Phi(r)=\frac{4 \pi}{72}<\alpha_{s}\left(G_{\mu \nu}^{a}\right)^{2}>\left(\rho^{-1}+\frac{3}{2} r^{-1} \alpha_{s}(r)\right)^{-1}
$$

and $\rho$ is the characteristic size of non-perturbative fluctuations dominating the gluon condensate, $<\alpha_{s}\left(G_{\mu \nu}^{a}\right)^{2}>$. Thus, we have the same $r^{2}$ correction in so far as

$$
<\alpha_{s}\left(G_{\mu \nu}^{a}\right)^{2}>\sim \Lambda_{Q C D}^{4}, \rho \sim \Lambda_{Q C D}^{-1}
$$

3. The examples given above indicate that it is the size of non-perturbative fluctuations which is crucial to determine the character of leading power corrections to the potential at short distances. What unifies renormalons and the model underlying Eq. (18), is that in both cases the characteristic size of non-perturbative fluctuations is of order $\Lambda_{Q C D}^{-1}$. To visualize the connection between the short and large distances in the most transparent way let us represent, in an Abelian case, the potential energy of the $Q \bar{Q}$ pair as an integral over space from the quark electric fields:

$$
V(r)=\frac{1}{4 \pi} \int d^{3} r^{\prime} \mathbf{E}_{1}\left(\mathbf{r}^{\prime}\right) \cdot \mathbf{E}_{2}\left(\mathbf{r}+\mathbf{r}^{\prime}\right) .
$$

In particular, the Coulomb potential can be obtained of course by integrating over the fields $\mathbf{E}_{1,2}$ of two point charges. On the other hand, if the electric fields are modified at large distances, then there arises a correction to the Coulomb energy at small distances as well.

Consider as an example two charges of opposite signs in a cavity of size $R$. Then the electric field of the charges, which is that of a dipole at large distances in empty space, changes at $r^{\prime}>R$. The corresponding change in $V(r)$ is of order

$$
\delta V(r) \sim \alpha r^{2} \int_{R}^{\infty} \frac{d^{3} r^{\prime}}{\left(r^{\prime}\right)^{6}} \sim \frac{\alpha r^{2}}{R^{3}}
$$

which is in agreement with the correction to the static potential discussed above. The renormalon-induced correction to the Coulombic potential (9) takes into account that at $r^{\prime} \geq \Lambda_{Q C D}^{-1}$ the fields are distorted by the confinement effects. The corresponding correction to the potential is of order

$$
\delta V(r) \sim r^{2} \int_{\Lambda_{Q C D}^{-1}}^{\infty} \frac{d^{3} r^{\prime}}{\left(r^{\prime}\right)^{6}} \sim r^{2} \Lambda_{Q C D}^{3}
$$

where $\alpha_{s}\left(r^{\prime}\right) \sim 1$ in this estimate.

From this point of view, it is not at all obvious that the major effect due to confinement is the modification of the Coulomb field of each of the quarks at $r^{\prime} \sim \Lambda_{Q C D}^{-1}$. Consider a simplified model according to which the electrostatic field of quarks is a 
correct zeroth-order aproximation only as far as it exceeds some critical value of order $\Lambda_{Q C D}^{2}$ :

$$
\left(\mathbf{E}^{a}\right)^{2} \sim \Lambda_{Q C D}^{4}
$$

while weaker fields do not penetrate the vacumm because of its specific, confining properties. From this condition we get an estimate of distances $R_{c r}$ where the electrostatic field of quarks is strongly modified:

$$
\frac{\alpha_{s} r^{2}}{R_{\mathrm{cr}}^{6}} \leq \Lambda_{Q C D}^{4}
$$

where for simplicity we have neglected the effect of the running of $\alpha_{s}\left(r^{\prime}\right)$.

The change in the potential is then of order:

$$
\lim _{r \rightarrow 0} \delta V \sim \frac{\alpha_{s} r^{2}}{R_{\mathrm{cr}}^{3}} \sim \alpha^{1 / 2} r \Lambda_{Q C D}^{2}
$$

i.e., we get a linear in $r$ leading correction to the potential at short distances. Note that a linear potential in the context of models of a stochastic vacuum has been claimed a long time ago [15], however, that derivation refers to large distances while the estimate (24) applies at short distances. It is worth emphasizing, that the estimate (24) is entirely dependent on the assumption (22) as applied to the dipole field of the $Q \bar{Q}$ pair. Similarly, the renormalon effect can be visualized as arising from a similar condition but applied to a Coulomb-like field of a quark. Since the field of a dipole is weaker than that of a charge, the critical value of $\mathbf{E}^{2}$ is reached at shorter distances and the feedback from these distances is stronger.

It is worth emphasizing that to realize condition (22) with $r \rightarrow 0$ one needs smallsize nonperturbative fluctuations in the QCD vacuum. Indeed $R_{\text {crit }} \rightarrow 0$ as $r \rightarrow 0$. This conclusion also fits well with the discussion above. That is why a confirmation of the existence of the linear correction (24) would provide very important insight into the mechanism of confinement. If one tries to speculate about what kind of fluctuations these could be, it is natural to turn to the dual superconductor picture of confinement [16] (for a recent review see [17]). Magnetic monopoles are a crucial field configuration in this case. The magnetic monopoles of QCD were introduced 18 in the Abelian projection of QCD where they appear as singular objects. Although this could be an artifact of the gauge fixing [18] convincing evidence for existence of monopoles as physical objects was obtained in just this gauge (see [19] and references therein). If the physical size of monopoles is indeed vanishing, the linear potential at large distances, or the area law for the Wilson loop, could well continue to $r \rightarrow 0$ where it becomes a correction to the Coulomb-like potential. Existing data on the lattice [11] do not indicate any change in the linear in $r$ piece of the potential at small distances but no special mesurements targeting this behaviour have been performed so far.

As is mentioned above, very recent measurements do indicate [10] the presence of a $1 / Q^{2}$ correction in the effective coupling ( see Eq(田) with a positive constant $c_{Q^{2}}$ :

$$
\left(c_{Q^{2}}\right)_{l a t}>0
$$

We observe that this sign is in fact opposite to what one would expect from a model in 
which the effective coupling at large distances is frozen 8$]$ :

$$
\alpha_{f r} \approx \frac{1}{b_{0}}\left(\frac{1}{\ln Q^{2} / \Lambda_{Q C D}^{2}}+\frac{\Lambda_{Q C D}^{2}}{\Lambda_{Q C D}^{2}-Q^{2}}\right) .
$$

On the other hand, if one assumes that linear in $r$ potential established at large distances continues to short distances as well then the $1 / Q^{2}$ correction to the coupling would have the same sign as indicated by the data (25). It is worth mentioning once more that although most recent discussions [0, 8] emphasize the analyticity aspect of the high- $Q^{2}$ behaviour of the coupling constant the analiticity itself does not fix the form of the correction (see, e.g., [20]). On the other hand, as is discussed above, the existence of small-size fluctuations seems to be a prerequisite for the $1 / Q^{2}$ corrections.

4. So far we discussed the static quark potential which can be measured on a lattice. Quark potential is also relevant to the physics of bound states of heavy quarks (for a review and references see [12]). As the zeroth order approximation one considers usually Coulomb-like states in the potential (1). Then if one treats the potential $K^{2} r$ as a perturbation the corrections to the energy levels are:

$$
\delta E_{n l}=\frac{1}{2}\left[3 n^{2}-l(l+1)\right] a K^{2}, \quad a=\frac{2}{m C_{F} \alpha}
$$

where $a$ is the corresponding Bohr radius. Nominally this correction is enhanced by powers of $m / \Lambda_{Q C D}$ as compared to correction due to the possible $r^{2}$ piece in the potential in (see Eq. (9)) or the Voloshin-Leutwyler correction [21]. In particular, the latter correction to energy levels is inversely proportional to $\mathrm{m}^{3}$ :

$$
\left(\delta E_{1}\right)_{V L}=\frac{1.67 \pi<\alpha_{s}\left(G_{\mu \nu}^{a}\right)^{2}>}{m^{3} C_{F}^{4} \alpha_{s}^{4}}
$$

where we have quoted the result for $n=1$ which might be the most relevant to phenomenological applications. There are, however, important caveats to this statement on the dominating role of the corrections due to the linear term, if it exists. First, standard calculations [22] already contain a linear term in the potential (extrapolated from large distances), so that $K^{2}$ shoud be understood rather as a deviation from this form of the potential at short distances. Furthermore, the Voloshin-Leutwyler correction contains large factors, like $\alpha^{-4}$ which are important numerically. Since we do not know yet of an estimate of the constant $K^{2}$, and also the value of the gluon condensate is not well known, it is difficult to compare the relative size of various contributions. The best strategy at the moment for the detection of the short-distance $K^{2} r$ piece is, as already stated, through lattice measurements.

5. On the theoretical side, the most important reservation about applying and comparing various corrections simultaneously is that the potential picture has a limited scope of validity. To clarify the applicability of the renormalon-induced and of the linear correction (see Eqs. (9) and (41), respectively) to quarkonium physics, let us first review the well known results from QED and QCD,

The particular QED effect which might imitate the interaction of quarks at short distances with large scale fluctuations is the shift of atomic levels in a cavity. In the static potential picture, the modification of the potential due to the electrostatic interaction of a dipole with the cavity, $\delta V(r) \sim r^{2} / R^{3}$, is found first (see Eq.(20). The energy 
shifts could be obtained then by averaging this potential over the unperturbed wave functions. This procedure would be equivalent to using the $r^{2}$ piece in the potential (9) as a perturbation on the Coulomb-like states.

A consistent quantum-mechanical treatment of the problem [23], on the other hand, starts with the dipole interaction:

$$
H_{\text {int }}=-e \mathbf{d} \cdot \mathbf{E} \text {. }
$$

where $\mathbf{E}$ is the electric field associated with zero-point fluctuations of the electromagnetic field in the cavity. The spectrum of the zero-point fluctuations depends on the size $R$ through the boundary condition, and one is interested in fact only in these $R$ dependent terms. Next, the shift in energy levels $E_{n}$ is obtained to second order in $H_{\text {int }}$ :

$$
\delta E_{n} \sim V_{n k}\left(E_{n}-E_{k}+\omega_{\text {char }}\right)^{-1} V_{k n} .
$$

We consider bound states and have set $V_{n k}=\left\langle n\left|\delta L_{i n t}\right| k\right\rangle$. Since we are interested only in estimates, we simply retain the contribution of a characteristic photon frequency, $\omega_{\text {char }} \sim 1 / R$. The result for $\delta E_{n}$ depends crucially on the relative magnitude of $E_{n} \sim$ $m \alpha^{2} \sim \alpha / a$ and $\omega_{\text {char }}$, i.e., on the relative magnitude of $R$ and $a / \alpha$, where $a$ is the Bohr radius. Note that a new scale, that is $a / \alpha$, emerges at this stage. Namely, if $\omega_{\text {char }} \gg E_{n}$ then

$$
\delta E_{0} \sim \alpha a^{2}\left(\mathbf{E}^{2}\right) R \sim \alpha \frac{a^{2}}{R^{3}}, \quad R \ll a / \alpha
$$

since $e^{2} \mathbf{d}^{2} \sim \alpha a^{2}$. The shift corresponds of course to evaluating the matrix element of the electrostatic potential, $\delta V(r) \sim \alpha r^{2} / R^{3}$ discussed above. Thus, in this case the potential picture does apply for the evaluation of the energy shifts.

On the other hand, if $R \gg a / \alpha$ then $\omega_{\text {char }}$ in the energy denominator of Eq. (30) can be neglected and

$$
\delta E_{0} \sim \alpha a^{2}(\mathbf{E})^{2} \frac{a}{\alpha} \sim \frac{a^{3}}{R^{4}}, \quad R \gg a / \alpha
$$

where we used $\mathbf{E}^{2} \sim R^{-4}$ for the characteristic frequencies. Eq. (32) is in clear violation of the potential picture. Eq (32) could be interpreted by saying that the electrostatic potential $\sim r^{2}$ is replaced, when the distance between the particles $a$ is much smaller than $\alpha R$, by an effective potential $\sim r^{3}$. But this is true only as far as rough estimates are concerned. Rigorously speaking, there is no potential whatsoever corresponding to the shifts obtained in this way [21]. Note also that the emergence of the scale $R \sim a / \alpha$ can be understood in a simple way as an effect of retardation. Indeed, the time needed to communicate with the distances of order of the size of cavity $R$ can be called the retardation time, $T_{r e t} \sim R$. For the potential picture to be valid this time should be smaller than the revolution time which is order, $T_{\text {rev }} \sim a / v \sim a / \alpha$. The potential picture becomes distorted once $T_{\text {ret }} \approx T_{\text {rev }}$.

In the QCD case one considers [21] atom-like systems of heavy quarks $Q$ with a size which is, at least formally, much smaller than the confinement radius,

$$
\alpha_{s}(M) \cdot M \gg \Lambda_{Q C D}
$$

where $M$ is the heavy quark mass. In this case the quarks are turning so fast and at such a small distance that they cannot be resolved by soft gluons. To account for the 
interaction of the $Q \bar{Q}$ pair with these soft gluons one starts again with the interaction:

$$
H_{\text {int }}=-\sqrt{\alpha_{s}}\left(t_{1}^{a}-t_{2}^{a}\right) \mathbf{d} \cdot \mathbf{E}^{a}
$$

where, the $t_{i}^{a}$ refer to the $Q$ and $\bar{Q}$ in the quarkonium, and by $\mathbf{E}^{a}$ one understands the soft gluonic fields (vaccum fields). While the exact form of these fields is an asyet unsolved strong-coupling problem, one assumes usually [1] that there is a typical frequency, $\omega_{\text {char }} \sim \Lambda_{Q C D}$. The intensity of the vacuum fields is characterized by a vacuum expectation value $\left\langle\alpha_{s}\left(G_{\mu \nu}^{a}\right)^{2}\right\rangle$ which is treated phenomenologically.

It may be worthwhile here to discuss the situation represented by Eq. (33) from the renormalon viewpoint. In order to do this we consider the shift in the interaction energy of the quarks in the quarkonium and look for the infrared sensitive contributions in perturbation theory. This is seen to arise from the class of diagrams shown in Fig.(1a). The external states are the color singlet quarkonium states, the exchanged gluon is soft (renormalon chain) and the intermediate state in this figure is a continuum color octet state which is short lived because of its high virtuality. Indeed the energy denominator for this intermediate state is $\sim(E-\epsilon) \sim B$, where $E$ is its energy, and $\epsilon$ that of the external color singlet quarkonium state with binding energy $B$. Since $B \gg \Lambda_{Q C D}$, (Eq.(33)), we see that the intermediate state is far off shell and we thus arrive at the reduced diagram shown in Fig (1b). The renormalon chain on the soft gluon line in this figure is the standard representation of the ( perturbative) gluon condensate $<\alpha_{s} G^{2}>$ which drives this contribution. A standard analysis ( see e.g., 223 ) produces a contribution proportional to $\Lambda_{Q C D}^{4} r^{3}$. It should be emphasized that the situation being discussed, i.e., $T_{r e v} \ll \Lambda_{Q C D}^{-1}$, is precisely the case when the potential picture is not valid for the non coulombic corrections.

Both the cases of static quarks [4, 5] and of atom-like systems (quarkonium) [21] with $E_{0} \gg \Lambda_{Q C D}$ were considered in the literature. In the latter case one arrives at a natural generalization of Eq. (32):

$$
\delta E \sim n^{6} \frac{\Lambda_{Q C D}^{4}}{\left(m \alpha_{s}\right)^{4}} m
$$

where we have indicated also the sharp dependence on the principal quantum number $n$. Note also that Eq. (35) has one extra power of $\alpha$ is the denominator as compared with (32) because it is the product $\alpha_{s}\left(G_{\mu \nu}^{a}\right)^{2}$ which is renormalization group invariant.

It is worth emphasizing also that in the QCD case there exists an extra problem with applying the potential picture to the bound $Q \bar{Q}$ states. Namely, the energies of intermediate states in Eq. (30) are now energies of color states since gluons carry color. Formally, these are $Q \bar{Q}$ continuum states. Color states are of course widely used in perturbative QCD, and in this respect the situation does not look exceptional. The standard constraint, however, is that color states can be excited only for short times and Eq. (30), when applied in QCD, is therefore consistent as long as the energy denominator is much larger than $\Lambda_{Q C D}$, i.e., in the situation discussed above.

These considerations suggest that, to be rigorous, the only case which can be treated consistently is when

$$
E_{n} \sim M \frac{\alpha_{s}(M)^{2}}{n^{2}} \gg \Lambda_{Q C D}
$$


This implies that it is not just the size of the system that has to be smaller than $\Lambda_{Q C D}$, but that the binding energy has to be large compared to $\Lambda_{Q C D}$. In the real world, where the condition is never satisfied by a wide margin, only a detailed calculation can tell the extent and degree of the applicability of the formalism. In this respect it would seem that reasonably good results may be obtained for $\bar{b} b$ with $n=1$ and, to a lesser extent, $n=2$ and $\bar{c} c$ with $n=1$ [12].

Returning to the case of the linear correction to the potential (41) we observe that for large enough quark masses, the retardation effects wipe out any kind of a potential so that the Voloshin-Leutwyler regime sets in. However, for the linear correction it happens at larger masses than for the quadratic correction since it is associated with shorter distances $R_{c r} \sim \alpha_{s}^{1 / 2} a^{1 / 3} \Lambda_{Q C D}^{-2 / 3}$ (see (23)) where $a$ is the Bohr radius. Therefore, the retardation and revolution times get comparable if

$$
a \leq \alpha^{9 / 4} \Lambda_{Q C D}^{-1}
$$

where $a$ is understood to be a function of the mass (and of the quantum number $n$ ). There is an extra power of $\alpha$ on the right hand side compared to the case of the standard correction.

9. To summarize, we have argued that the assumption that weak color fields do not penetrate the vacuum, implies a linear correction to the static quark potential at short distances. For this hypothesis to be realized there should exist small-size nonperturbative fluctuations. If these fluctuations are monopoles then the linear potential at large distances could possibly be extrapolated to short distances which does not contradict any (lattice) data at the moment. The recent indication [10] that the running coupling has a $1 / Q^{2}$ correction does imply a linear correction to the potential as well. If confirmed, therefore, it would indicate the existence of small-size non-perturbative fluctuations. We have also considered phenomenological implications of the linear correction to the potential for quarkonia but found that at this time no conclusion can be drawn on the existence or absence of such a correction.

Acknowledgements. Part of this work was done in April 1995 when we were visiting the University Autonoma de Madrid. We are grateful to the Departamento de Física Teórica there for hospitality. We would like to thank F. J. Ynduráin for an initial collaboration. We have benefitted from interesting discussions with G. Marchesini, A. Vainshtein, P. van Baal. 


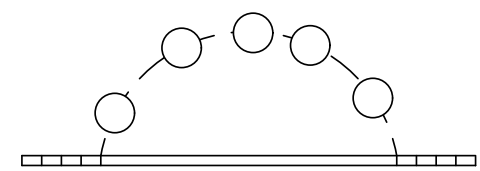

Fig. 1a Class of diagrams contributing to the shift in interaction energy of heavy quarks in quarkonium

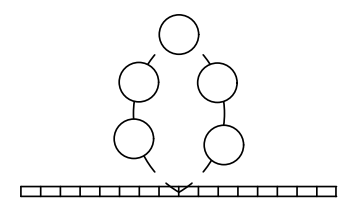

Fig. 1b Reduced diagram for far off-shell intermediate states. 


\section{References}

[1] M. A. Shifman, A. I. Vainshtein and V. I. Zakharov, Nucl. Phys. B147 (1978) 385.

[2] G. 't Hooft, in 'The Whys of Subnuclear Physics', Proc. Int. School, Erice, 1977, ed. A. Zichichi (Plenum, New York, 1978);

B. Lautrup, Phys. Lett. B69 (1977) 109.

[3] R. Akhoury and V. I. Zakharov, Nucl. Phys. Proc. Suppl. 54A (1997) 217;

M. Beneke, hep-ph/9706457.

[4] I.I. Balitsky, Nucl.Phys. B254 (1983) 166.

[5] H. G. Dosch, Phys. Lett. B 190 (1987), 177;

H. G. Dosch and Yu. A. Simonov, Phys. Lett. B205 (1988), 339;

Yu. A. Simonov, S. Titard and F.J. Ynduráin, Phys. Lett., B354 (1995) 435.

[6] U. Aglietti and Z. Ligeti, Phys. Lett., B364 (1995) 75

[7] G. Grunberg, hep-ph/9705290, hep-ph/9705460

R. Akhoury and V. I. Zakharov, hep-ph/9705318.

[8] D.V. Shirkov and I.L. Solovtsev, hep-ph/9704333; D.V. Shirkov, hep-ph/9708480.

[9] V.I. Zakharov, Nucl. Phys. 385 (1992) 385;

A. I.Vainshtein and V. I. Zakharov, Phys. Rev. Lett. 73 (1994) 1207; Phys. Rev. D54 (1996) 4039;

K.K. Yamawaki and V. I. Zakharov, hep-ph/9406373.

[10] G. Burgio, F. Renzo, G. Marchesini, and E. Onofri, hep-ph/9706209

[11] G. Bali, K. Schilling and A. Wachter, hep-lat/9506017

[12] F.J. Ynduráin, hep-ph/9708448; S Titard and F. J. Ynduráin, Phys Rev. D49 (1994), 6007 and D51 (1995), 6348.

[13] M. Peter, Phys. Rev. Lett., 78 (1997) 602.

[14] A.H. Mueller, Nucl.Phys., B250 (1985) 327.

[15] H.G. Dosch, Phys. Lett. B190 (1987) 177.

[16] G. 't Hooft, in "High Energy Physics", EPS International Conference, Palermo 1975, ed. A. Zichichi.

S. Mandelstam, Phys. Rep. 23C (1976) 245.

[17] M. Baker, hep-ph/9609269:

A. Di Giacomo, hep-lat/9606001.

[18] G. 't Hooft, Nucl. Phys. B190 (1981) 455.

[19] M.I. Polikarpov, Nucl.Phys. Proc. Suppl. 53 (1997) 134;

B.L.G. Bakker, M.N. Chernodub and M.I. Polikarpov, hep-lat/9706007.

[20] Yu. L. Dokshitzer, G. Marchesini and B.R. Webber, Nucl. Phys. B469 (1996) 93. 
[21] Voloshin, Nucl. Phys. B154 (1979) 155; Sov. J. Nucl. Phys. 36 (1982) 143.

H. Leutwyler, Phys. Lett. B98 (1981) 447.

[22] See e.g., J. Pantaleone, S.-H. Tye and Y. J. Ng, Phys. Rev. D33,777 (1986); F. Halzen et al., Phys. Rev., D47, 3013 (1993).

[23] H.B.G. Casimir and D. Polder, Phys.Rev. 73 (1948) 360.

C. Itzykson and J.-B. Zuber, Quantum Field Theory, McGraw Hill, (1980). 